\title{
IMÁGENES DE UN AMELOBLASTOMA: DE LA RADIOGRAFÍA A LA TOMOGRAFÍA
}

\section{IMAGES OF AMELOBLASTOMA: FROM RADIOGRAPHY TO TOMOGRAPHY}

\author{
TITULO CORTO: IMÁGENES DE UN AMELOBLASTOMA
}

\author{
Raúl Herrera-Mujica ${ }^{1,2}$, Andrés Agurto-Huerta ${ }^{1,2}$, León Palti-Menéndez ${ }^{1,2}$ y \\ Yazmín Pariamachi-Polino, ${ }^{1,2}$
}

Recibido en febrero 13 de 2015

Aceptado en mayo 07 de 2015

\section{RESUMEN}

Los tumores odontogénicos forman un grupo de lesiones de los maxilares que se originan en alguna alteración del patrón normal de la odontogénesis. Dentro de ellos se tiene al ameloblastoma, que es probablemente el de mayor controversia en cuanto a su manejo, dada su conducta local agresiva, su elevada tasa de recurrencia y su pequeña probabilidad de malignización. Las radiografías cumplen un papel importante en la evaluación de este tumor, pero su principal limitación es que son estudios por imágenes en dos dimensiones. La tomografía computarizada de haz cónico (cone beam) es una valiosa herramienta que permite una exhaustiva observación de las estructuras anatómicas, gracias a la posibilidad de efectuar cortes en diferentes planos del espacio; todo esto debido a las diferentes opciones de visualización que proporciona. Por consiguiente, la evaluación del ameloblastoma es mucho más completa y permite analizar los tejidos óseos sin distorsión ni magnificación.

Palabras clave: Ameloblastoma; radiografía; tomografía. (fuente DeSC)

\section{Abstract}

Odontogenic tumors are a group of lesions originated from odontogenesis course alterations. Within odontogenic tumors is ameloblastoma, its treatment probably is one of the most controversial, because its aggressive behavior, high recurrence and probably low malignancies. Radiographs play an important role in assessment from this tumor, but the limitation is they are in two dimensions exams. Cone beam computed tomography is a valuable tool because enable a comprehensive assessment of anatomical structures by multiple sections of the area. Therefore, evaluation of ameloblastoma is complete by cone beam computed tomography because bone structures do not show distortion neither magnification.

Keywords: Ameloblastoma, radiography, tomography. (fuente MeSH)

\footnotetext{
1. Especialista en Radiología Oral y Maxilofacial egresado de la Universidad Peruana Cayetano Heredia.

2. Miembro de la Asociación Peruana de Radiología Bucal y Maxilofacial (APERBUM) y Miembro del staff de radiólogos del Instituto de Diagnóstico Maxilofacial (IDM), Lima - Perú. Correos electrónicos: dr_herrera@idmperu.com; dr_agurto@idmperu.com; dr_palti@idmperu.com; dra_pariamachi@idmperu.com
} 


\section{INTRODUCCIÓN}

$\mathrm{E}^{\mathrm{l}}$ ameloblastoma es uno de los tumores benignos más frecuentes del complejo maxilofacial, se le ha descrito principalmente en los sectores posteriores de la mandíbula, ya sea en la zona de la rama, del cuerpo y/o ángulo, con una frecuencia del $80 \%$ de presentación en la mencionada región. En cuanto al sexo y a la edad, afecta por igual a hombres y mujeres, con una mayor prevalencia entre la tercera y la cuarta década de vida. Esta neoplasia comprende diferentes manifestaciones clínicas, radiográficas e histopatológicas, y a pesar de que se trata de una patología benigna es localmente invasora y recidiva con bastante facilidad ${ }^{1}$. El ameloblastoma es de origen epitelial sin inducción en el tejido conectivo. Para el tratamiento, si bien es cierto, es muy importante un adecuado estudio por imágenes, es trascendental evaluar los signos y síntomas clínicos, el tamaño, la localización, la edad del paciente y el tipo histológico de la entidad².

\section{Estudio Radiológico}

La radiografía es la representación bidimensional de una estructura tridimensional. Se trata de imágenes analógicas, es decir, representaciones análogas o semejantes a los objetos o estructuras reales. Una radiografía muestra los diferentes grados de atenuación que sufre un haz de rayos $\mathrm{X}$ al atravesar un cuerpo ${ }^{3}$.

Las diferentes técnicas radiográficas desempeñan una labor trascendental en la evaluación del ameloblastoma, ya que proporcionan valiosa información en cuanto a su densidad, extensión, límites, su posible asociación a alguna pieza dentaria y el compromiso que ocasiona a las estructuras óseas vecinas. La radiografía panorámica es la técnica apropiada para evaluar de manera preliminar las características de esta lesión y está a disposición del odontólogo desde hace ya muchos años, permitiendo observar todas las estructuras dentarias y maxilofaciales en una imagen plana; pero estas mismas estructuras se muestran de forma distorsionada y con un aumento variable de tamaño $0^{4,5}$.

\section{Tomografía Computarizada de haz cónico (Cone Beam)}

La tomografía computarizada de haz cónico es un registro diagnóstico que en la actualidad promete una gran precisión dimensional sin ningún tipo de superposición de las estructuras anatómicas. El volumen tridimensional del cuerpo a ser estudiado es adquirido a través de un solo barrido de escáner; es justamente esta particularidad la que lo diferencia de la imagen de la tomografía espiral multicorte (TEM). La tomografía computarizada de haz cónico se basa en la relación simple y directa entre el sensor 2D y la fuente de radiación, los cuales rotan sincrónicamente alrededor de la cabeza del paciente. La forma del haz de rayos X es cónica y obtiene un volumen de datos esférico o cilíndrico conocido como campo de visión o field of view (FOV) ${ }^{6,7}$.

Por medio de la tomografía computarizada de haz cónico se pueden generar imágenes seccionales y tangenciales del hueso alveolar, además de imágenes tridimensionales del arco dentario. Proporciona también cortes secuenciales a lo largo del reborde alveolar en intervalos que pueden ser determinados a voluntad. Se puede visualizar el reborde alveolar en tres dimensiones y medir la altura de hueso en un área determinada ${ }^{8,9}$. Una de las opciones que ofrece el software de la tomografía computarizada de haz cónico es la reconstrucción 3D, que muestra la región en estudio en tres dimensiones y proporciona una visión bastante ilustrativa de la forma y el volumen del tejido óseo?

\section{Presentación imaginológica de un caso}

A continuación se presenta el caso de una paciente de 16 años de edad que acudió a la consulta por presentar asimetría facial a consecuencia de un aumento de volumen en el lado izquierdo de la mandíbula. A la evaluación de la radiografía panorámica se observa una lesión radiolúcida unilocular de límites definidos; bordes parcialmente corticalizados; localizada en el cuerpo, ángulo y tercio inferior de la rama ascendente

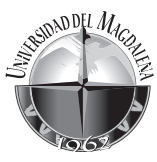


mandibular del lado izquierdo. La mencionada lesión ocasiona la reabsorción radicular externa de la pieza 36; el desplazamiento cefálico y distal de la pieza 38; y caudal de la pieza 37. Así mismo, se evidencia el borramiento parcial de la línea oblicua externa; desplazamiento caudal del conducto dentario inferior y adelgazamiento del reborde basal (Figura 1).

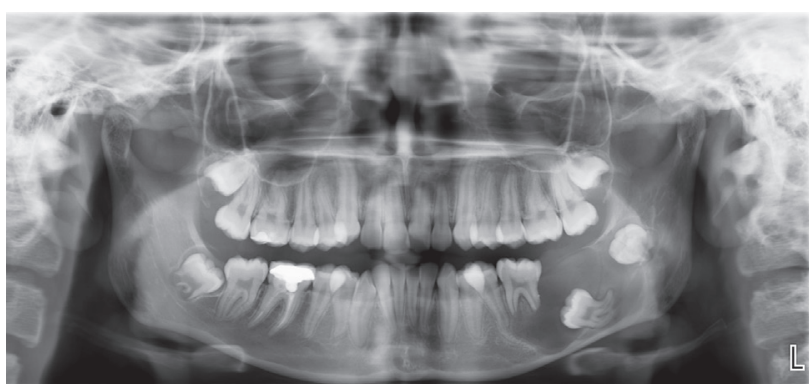

Figura 1. Radiografía Panorámica

El software que ofrece la tomografía computarizada de haz cónico da la posibilidad de evaluar la lesión desde las tres dimensiones del espacio mediante la opción de la reconstrucción multiplanar (MPR); la cual muestra la extensión de la lesión isodensa en reconstrucciones axiales, coronales y sagitales. La lesión condiciona el desplazamiento, adelgazamiento y erosión de las tablas óseas; tanto vestibular como lingual (Figura 2).
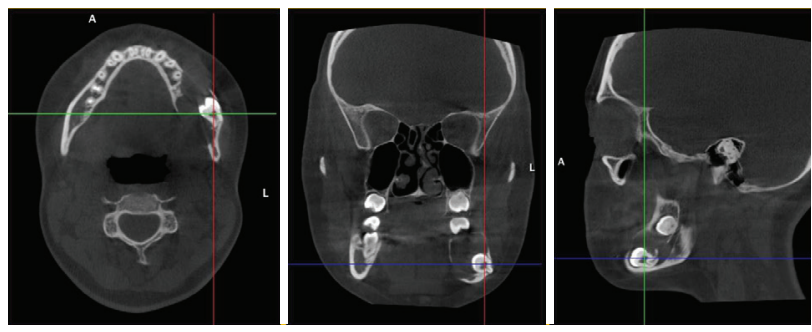

Figura 2. Reconstrucción Multiplanar. Vista axial (imagen izquierda). Vista coronal (imagen del centro). Vista sagital (imagen derecha)

Otra posibilidad que ofrece la tomografía computarizada de haz cónico es la reconstrucción panorámica, que es una imagen bastante parecida a la de la radiografía panorámica. Esta reconstrucción facilita al cirujano dentista la evaluación de las características de la lesión por su semejanza a la técnica radiográfica antes mencionada (Figura 3).

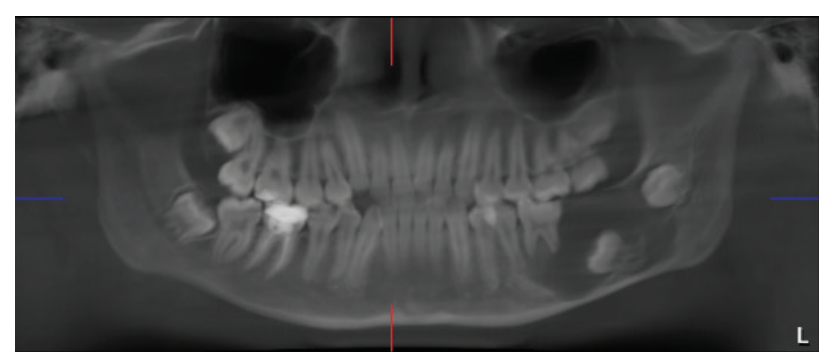

Figura 3. Reformación Panorámica

En base a la reconstrucción panorámica se realiza la planificación de los cortes transaxiales, los cuales toman en cuenta la forma de la arcada dentaria y son ideales para evaluar de manera más exacta la verdadera extensión de la lesión (Figura 4).

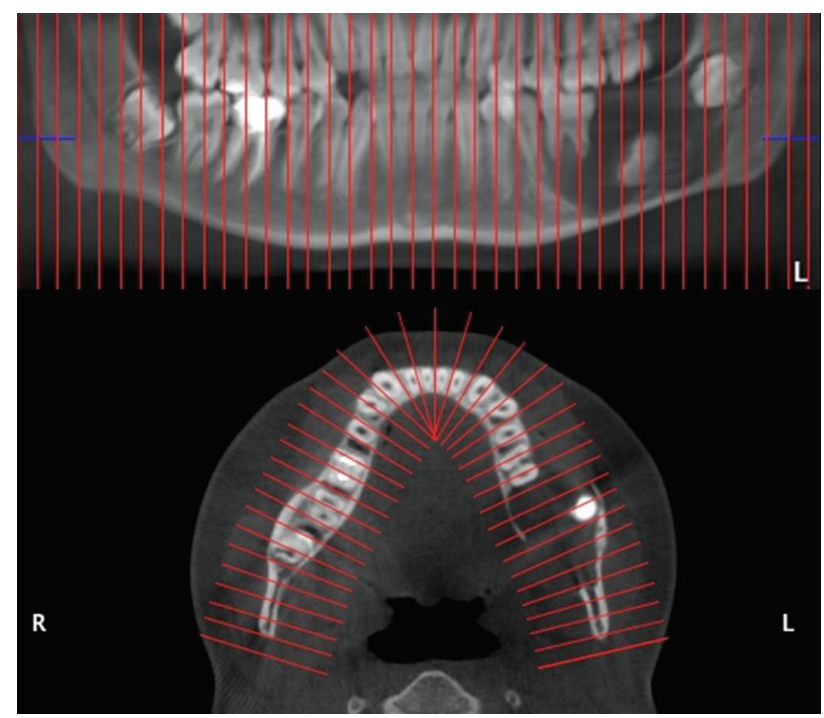

Figura 4. Distribución de los cortes transaxiales

Se procede a delimitar el trayecto del conducto dentario inferior, el mismo que se encuentra desplazado en dirección caudal y que está en estrecha relación con el reborde basal y con la pieza 37, esta última se encuentra en estrecha relación con la tabla ósea vestibular. Así mismo, en los cortes transaxiales a nivel de la pieza 37, se observa la erosión de las tablas óseas (Figura 5). 


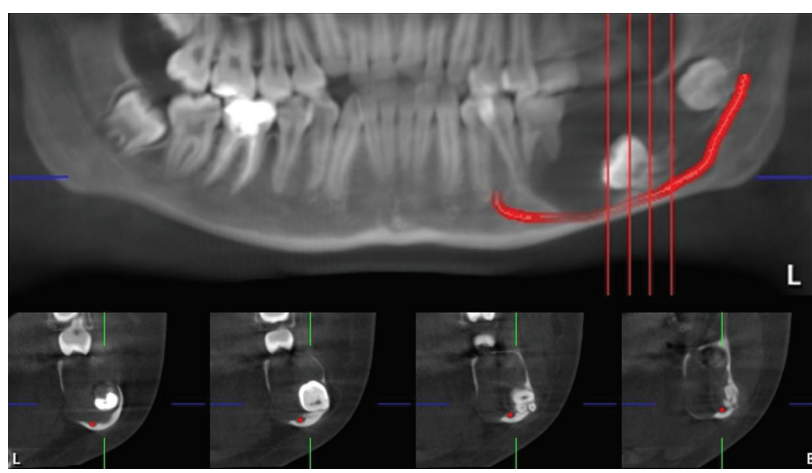

Figura 5. Delimitación del conducto dentario inferior

La reconstrucción en 3D representa a la lesión de una manera mucho más ilustrativa y comprensible; pudiendo observar la lesión desde distintas perspectivas, apreciando su relación con las estructuras anatómicas vecinas (Figura 6).

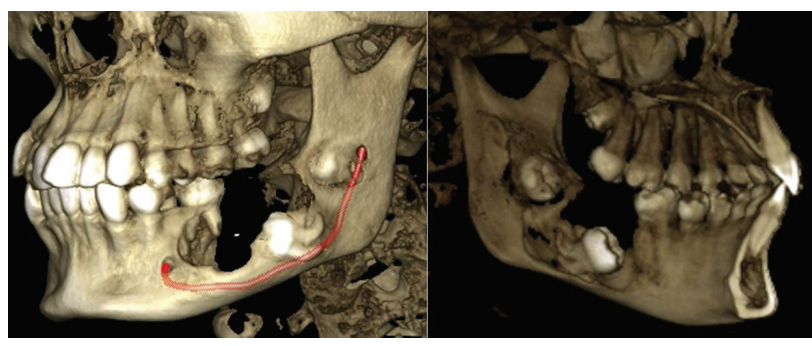

Figura 6. Reconstrucciones en 3D

Se debe considerar que la presentación de este caso es con el propósito de describir las espléndidas herramientas que ofrece la tomografía computarizada de haz cónico (cone beam), en comparación con los estudios 2D, cuando se realiza el estudio por imágenes de las distintas patologías que comprometen el tejido óseo en la región bucomaxilofacial. Tomando en cuenta todas las características imaginológicas anteriormente mencionadas, se concluye que se trata de una lesión tumoral benigna compatible con ameloblastoma.

\section{DISCUSIÓN}

El ameloblastoma es un tumor benigno proveniente de tejido odontogénico, localmente agresivo e invasivo, de crecimiento lento, pero persistente ${ }^{10}$. La localización es un aspecto importante a considerar. Esta entidad se localiza en un $80 \%$ en la mandíbula y en un $20 \%$ en el maxilar. A nivel de la mandíbula, el 70 \% se presentan en la región molar o rama ascendente, el 20 \% en la región premolar y un $10 \%$ en la región anterior. A nivel del maxilar se puede mencionar que este tumor se localiza en un $47 \%$ en la región molar, $15 \%$ en las regiones del seno y piso de fosa nasal, $9 \%$ en la región canina y $2 \%$ en el paladar. Se debe considerar también que el 10 al 15\% de los tumores se asocian a una pieza dentaria no erupcionada ${ }^{11}$.

Las técnicas de diagnóstico por imagen nos encaminan a un plan de tratamiento coherente, ya que permiten definir la decisión operatoria a ser asumida debido a que confirma las hipótesis diagnósticas del examen clínico ${ }^{12}$.

La exploración radiográfica tiene la finalidad de proporcionar la topografía de la región anatómica que será evaluada en la región maxilofacial; más aún el odontólogo debe saber que hoy en día éstas técnicas en 2D dejaron de ser las más adecuadas. Entre las distintas técnicas radiográficas se tiene a la radiografía panorámica u ortopantomografía que presenta una distorsión por amplitud entre los reparos anatómicos radiografiados y la imagen resultante. Este inconveniente conlleva al aumento del tamaño de la imagen en relación a las estructuras de interés, ocasionando entonces una limitación en la confiabilidad dimensional de la mencionada técnica ${ }^{13}$.

Radiográficamente, el ameloblastoma se observa como una imagen osteolítica con límites definidos, bordes corticalizados y puede estar o no asociado con piezas dentarias no erupcionadas. Su presentación es variable: unilocular, multilocular o pseudolocular; semejando de esta manera una apariencia de pompas de jabón o panal de abejas. Las piezas dentarias comprometidas por la lesión suelen estar vitales y posteriormente suelen ser desplazadas, presentar reabsorción radicular y movilidad ${ }^{1}$.

Es de conocimiento que las imágenes en 2D tradicionales, como la radiografía panorámica, son susceptibles a distorsión y magnificación al momento de evaluar su extensión y dimensiones. Las imágenes obtenidas mediante la tomografía computarizada de haz cónico ofrecen una óptima y más acertada posibilidad del diagnóstico y planeación del tratamiento, puesto que los reparos anatómicos del complejo maxilofacial están reproducidos en su forma y tamaños verdaderos. Existen varios tipos de imágenes que se pueden generar en base a la información recabada por esta técnica imaginológica (cone beam), tales como cortes reformateados multiplanares, proyecciones cefalométricas frontales y laterales en 2D, imágenes volumétricas y reconstrucciones panorámicas ${ }^{14}$. 
Hoy en día nuestra especialidad está cursando por un cambio extraordinario, debido a la incorporación de la sofisticada tecnología de la tomografía computarizada de haz cónico, la misma que posee distintas denominaciones, como tomografía cone beam, tomografía dental 3DX, cone beam tomography, CT Dental 3D, tomografía CB o tomografía volumétrica. Un elemento fundamental a favor de la tecnología Cone Beam es que utiliza muy bajas dosis de radiación para obtener imágenes de alta calidad, siendo la misma 15 veces menor que la tomografía espiral multicorte ${ }^{15}$.

\section{CONCLUSIONES}

La tomografía computarizada de haz cónico (cone beam) es una herramienta diagnóstica fundamental que permite evaluar las distintas estructuras del macizo facial. Es de significativa importancia para la evaluación de entidades patológicas de los maxilares, como quistes y tumores; ya que en las imágenes obtenidas se determina con precisión la zona que interesa explorar.

El odontólogo de práctica general debe tener la capacidad de reconocer y diagnosticar las patologías orales más frecuentes. Así mismo, está en la obligación de capacitarse y actualizarse adecuadamente en el uso de las nuevas tendencias del diagnóstico por imagen en el campo de la estomatología.

\section{AGRADECIMIENTOS}

Al Instituto de Diagnóstico Maxilofacial (IDM) por la gentileza de proporcionar las imágenes para el presente artículo.

\section{REFERENCIAS BIBLIOGRÁFICAS}

1. Duque FL, Radi JN, Del Valle AF. Ameloblastoma: reporte de diez casos y revisión de la literatura. Rev Fac Odont Univ Ant. 2003; 15 (1): 57-66.
2. Torres D, Infante P, Hernández JM, Gutiérrez JL. Mandibular ameloblastoma. A review of the literature and presentation of six cases. Med Oral Patol Oral Cir Bucal 2005;10: 231-8.

3. Cabrero FJ. Imagen Radiológica. Principios físicos e instrumentación. 1ra ed. Barcelona: Masson; 2004.

4. Urzúa R. Técnicas Radiográficas Dentales y Maxilofaciales. Aplicaciones. 1ra ed. Antioquía: Amolca; 2005.

5. Duterloo HS. Atlas de la Dentición Infantil. Diagnóstico ortodóncico y radiología panorámica. 1ra ed. Barcelona: Labor; 1992.

6. Lenguas AL, Ortega R, Samara G, López MA. Tomografía computarizada de haz cónico. Aplicaciones clínicas en odontología; comparación con otras técnicas. Cient dent. 2010; 7(2): 147-159.

7. Macleod I, Heath N. Cone- Beam Computed Tomography (CBCT) in dental practice. Dent Update. 2008; 35: 590-598.

8. White SC, Pharoah MJ. Oral Radiology: principles and interpretation. 5ta ed. St. Louis: Mosby; 2007.

9. Scarfe WC, Farman AG. What is Cone-Beam CT and How Does it Work? The Dental Clinics of North America. 2008; 52: 707-730.

10. Riascos R, Figueroa R, Martínez F, Martínez S, Borrero L. Revista Colombiana de Radiología. 2005; 16 (4): 1836-42.

11. Morales D. Ameloblastoma. Revisión de la literatura. Revista Cubana de Estomatología. 2009; 46(3): 48-61.

12. Fernandez-Formoso N, et al. Technical report. A paralleling technique modification to determine the bone crest level around dental implants. Dentomaxillofacial Radiology. 2011; 40: 385-389.

13. Rojas F, Figueroa M, Díaz V. Estudio Comparativo de la Especificidad Dimensional del Cone Beam y la Radiografía Panorámica Digital. Rev. Clin. Periodoncia Implantol. Rehábil. Oral. 2009; 2(3); 157-160.

14. Afrashtefar KI. Utilización de Imagenología bidimensional y tridimensional con fines odontológicos. Revista ADM. 2012; 69(3): 114-119

15. Aranyarashkul P, Caruso J, Gantes B, Schulz E, Riggs M, Dus I, et al. Bone density assessments of dental implant sites: Quantitative cone-beam computadorized tomography. Int J Oral Maxillofac Implants. 2005; 20(3):416-24.

Para citar este artículo: Herrera-Mujica R, Agurto-Huerta A, Palti-Menéndez L, PariamachiPolino Y. Imágenes de un ameloblastoma: de la radiografía a la tomografía. Duazary. 2015 dic; 12 (2): $182-186$ 